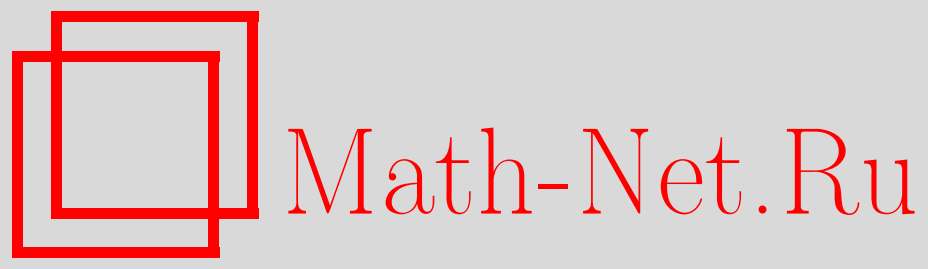

Э. Б. Винберг, Пределы интегрируемых гамильтонианов на полупростых алгебрах Ли, Функи. анализ и его прил., 2014, том 48, выпуск 2, 39-50

DOI: https://doi.org/10.4213/faa3143

Использование Общероссийского математического портала Math$\mathrm{Net.Ru}$ подразумевает, что вы прочитали и согласны с пользовательским соглашением

http://www . mathnet.ru/rus/agreement

Параметры загрузки:

IP : 54.237 .59 .107

26 апреля 2023 г., 14:45:12

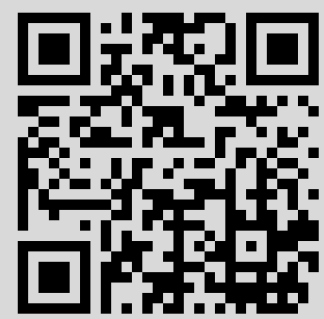


Функиионалъный анализ и его приложения

2014, т. 48, вып. 2, с. 39-50

УДК 512.81

\title{
Пределы интегрируемых гамильтонианов на полупростых алгебрах Ли*
}

\author{
(C) 2014. Э. Б. Виньерг
}

Посвящается Израилю Моисеевичу Гельфанду в связи с его столетием

\begin{abstract}
Мы доказываем, что предел интегрируемых гамильтонианов на полупростой алгебре Ли также является интегрируемым гамильтонианом, и строим некоторые пределы интегрируемых гамильтонианов, получаемых методом сдвига инвариантов, которые сами таким методом получены быть не могут.
\end{abstract}

Пусть $\mathfrak{g}$ - полупростая алгебра Ли и $P(\mathfrak{g})$ - соответствующая алгебра Пуассона, т. е. симметрическая алгебра пространства $\mathfrak{g}$, снабженная стандартной скобкой Пуасссона. Отождествляя при помощи инвариантного скалярного умножения алгебру $\mathfrak{g}$ с ее дуальным пространством $\mathfrak{g}^{*}$, мы будем рассматривать элементы алгебры $P(\mathfrak{g})$ как полиномиальные функции на $\mathfrak{g}$.

Метод сдвига инвариантов, восходящий к Манакову [2] и сформулированный и обоснованный в общем виде в работе Мищенко и Фоменко [3], связывает с каждым регулярным элементом $a \in \mathfrak{g}$ некоторую коммутативную (в смысле скобки Пуассона) однородную подалгебру $C(a)$ алгебры $P(\mathfrak{g})$ максимальной возможной степени трансцендентности (равной размерности борелевской подалгебры алгебры $\mathfrak{g})$. Подалгебра $C(a)$ свободно порождается базисными инвариантами присоединенной группы алгебры $\mathfrak{g}$ и их ненулевыми кратными производными по направлению $a$. Коммутативные подалгебры такого вида мы будем называть подалгебрами Мищенко-Фоменко.

Если элемент $a$ стремится к сингулярному элементу $a_{0}$ таким образом, что подалгебра $C(a)$ стремится к некоторому пределу, то этот предел будет коммутативной однородной подалгеброй с тем же рядом Пуанкаре. Степень трансцендентности предельной подалгебры совпадает с ее размерностью ГельфандаКириллова и определяется ее рядом Пуанкаре (см. об этом подробнее ниже). Следовательно, она совпадает со степенью трансцендентности алгебры $C(a)$. Мы также приводим иное доказательство этого факта, дающее алгоритм нахождения базиса трансцендентности предельной подалгебры.

Ситуация, когда элемент $a$ стремится к $a_{0}$, оставаясь в фиксированной картановской подалгебре, была детально исследована в работах автора [1] и Шувалова [4]. В частности, в последней работе было доказано, что в этой ситуации предельная подалгебра всегда свободна и имеет ту же степень трансцендентности, что и $C(a)$. Вопрос о том, не будет ли всегда предельная подалгебра свободной (или хотя бы конечно порожденной), остается открытым.

\footnotetext{
*Работа была выполнена при поддержке гранта РФФИ 12-01-00704.
} 
Мы приведем некоторые примеры пределов подалгебр Мищенко-Фоменко, отличные от тех, которые были рассмотрены в [1], [4], и обсудим проблему описания многообразия всех интегрируемых квадратичных гамильтонианов, получаемых таким путем.

Данная работа была завершена во время моего пребывания в университете Билефельда в мае-июне 2013 г. Я благодарю этот университет за гостеприимство.

\section{§1. Формальные пределы однородных подалгебр алгебры многочленов}

Предел последовательности однородных подалгебр алгебры многочленов над $\mathbb{R}$ или $\mathbb{C}$, имеющих один и тот же ряд Пуанкаре, может естественным образом пониматься как сумма пределов их однородных компонент в смысле соответствующих грассмановых многообразий. Нам, однако, будет удобнее, несколько сузив это понятие предела, формализовать его, заменив последовательность однородных подалгебр одной подалгеброй в алгебре многочленов над полем формальных рядов Лорана.

Пусть $K$ - некоторое поле. Через $K[t], K[[t]]$ и $K((t))$ мы будем, как обычно, обозначать алгебры многочленов и формальных степенных рядов и поле формальных рядов Лорана с коэффициентами из $K$.

Для всякого векторного пространства $V$ над полем $K$ положим

$$
V[t]=V \otimes K[t], \quad V[[t]]=V \otimes K[[t]], \quad V((t))=V \otimes K((t)) .
$$

Отметим, что $V((t))$ - векторное пространство над полем $K((t))$ той же размерности, что и $V$ над полем $K$. Обозначим через v канонический гомоморфизм $V[[t]] \rightarrow V$ («взятие значения в нуле»).

Определение 1. Формальным пределом (при $t \rightarrow 0$ ) подпространства $U(t)$ пространства $V((t))$ называется подпространство

$$
U(0)=\mathfrak{v}(U(t) \cap V[[t]])
$$

пространства $V$.

Если пространство $U(0)$ конечномерно, то любые прообразы его базисных векторов в $U(t) \cap V[[t]]$ составляют базис пространства $U(t)$, так что

$$
\operatorname{dim} U(0)=\operatorname{dim} U(t) .
$$

В общем случае прообразы линейно независимых векторов пространства $U(0)$ линейно независимы, так что если пространство $U(0)$ бесконечномерно, то и пространство $U(t)$ бесконечномерно.

Снабдим пространство $V((t))$ формальной топологией, приняв за окрестности нуля подмножества вида $t^{m} V[[t]], m \in \mathbb{Z}$. Из соображений непрерывности ясно, что если подпространство $U(t)$ конечномерно, его формальный предел не изменится при замене базисных векторов достаточно близкими им векторами.

Пусть теперь $A=\bigoplus_{m=0}^{\infty} A_{m}-$ градуированная алгебра с $A_{0}=K$ и $\operatorname{dim} A_{m}=$ $d_{m}(A)<\infty$ при всех $m$. Ее рядом Пуанкаре называется формальный степенной ряд

$$
P(A, z)=\sum_{m=0}^{\infty} d_{m}(A) z^{m} .
$$


В дальнейшем мы будем считать, что $A$ - целостная алгебра, т. е. коммутативная ассоциативная алгебра без делителей нуля.

Если $A$ - конечно порожденная алгебра, то ее ряд Пуанкаре есть ряд Тейлора некоторой рациональной функции, голоморфной в круге $|z|<1$ и имеющей в 1 полюс порядка, равного степени трансцендентности $\operatorname{tr} . \operatorname{deg} A$ алгебры $A$; тем самым степень трансцендентности алгебры $A$ определяется ее рядом Пуанкаре.

В любом случае степень трансцендентности алгебры $A$ совпадает с ее размерностью Гельфанда-Кириллова $\operatorname{Dim} A[6] . \mathrm{B}$ [5, теорема 4.5] доказано, что если алгебра $A$ вкладывается в конечно порожденную градуированную алгебру (в качестве градуированной подалгебры), то

$$
\operatorname{Dim} A=\limsup _{m \rightarrow \infty} \frac{\log \sum_{k=0}^{m} d_{k}(A)}{\log m} .
$$

Тем самым и в этом случае степень трансцендентности алгебры $A$ определяется ее рядом Пуанкаре.

Если $B(t)=\bigoplus_{m=0}^{\infty} B_{m}(t)\left(B_{m}(t) \subset A_{m}((t))\right)$ - однородная подалгебра $K((t))$ алгебры $A((t))=\bigoplus_{m=0}^{\infty} A_{m}((t))$, то формальный предел $B(0)=\bigoplus_{m=0}^{\infty} B_{m}(0)$ будет однородной подалгеброй алгебры $A$ с тем же рядом Пуанкаре, что и $B(t)$.

В дальнейшем предполагается, что

$$
A=K\left[x_{1}, \ldots, x_{n}\right]
$$

- алгебра многочленов со стандартной градуировкой. Алгебра $A((t))$ может рассматриваться как алгебра формальных рядов Лорана с коэффициентами из $A$ или как алгебра многочленов над полем $K((t))$.

Если $K$ - поле нулевой характеристики, то многочлены $f_{1}(t), \ldots, f_{d}(t) \in$ $A((t))$ алгебраически независимы тогда и только тогда, когда их якобиева матрица имеет ранг $d$ (как матрица с элементами из $A((t)))$. Отсюда следует, что в пространстве всех наборов из $d$ однородных многочленов заданных степеней алгебраически независимые наборы образуют подмножество, открытое в формальной топологии.

Предложение 1. Предположим, что $K$ - поле нулевой характеристики $u$ подалгебра $B(t) \subset A((t))$ свободно порожсдается однородными многочленами $f_{1}(t), \ldots, f_{d}(t) \in A((t))$, причем предельная подалгебра $B(0) \subset A$ является конечно порожденной. Тогда любые однородные многочлены $\tilde{f}_{1}(t), \ldots, \tilde{f}_{d}(t) \in$ $A((t))$, достаточно близкие $\kappa f_{1}(t), \ldots, f_{d}(t)$, свободно порожсдают подалгебру $\widetilde{B}(t)$, имеющую тот же формальный предел, что и $B(t)$.

Доказательство. Из сказанного выше следует, что если многочлены $\tilde{f}_{1}(t)$, $\ldots, \tilde{f}_{d}(t)$ достаточно близки к $f_{1}(t), \ldots, f_{d}(t)$, то они алгебраически независимы и, следовательно, подалгебры $\widetilde{B}(t)$ и $B(t)$, а значит, и их предельные подалгебры $\widetilde{B}(0)$ и $B(0)$ имеют один и тот же ряд Пуанкаре. Далее, любой однородный элемент подалгебры $B(0)$ есть по определению свободный член некоторого формального степенного ряда из $A[[t]]$, представимого в виде $F\left(f_{1}(t), \ldots, f_{d}(t)\right)$, где $F$ - многочлен с коэффициентами из $K((t))$. Если многочлены $\tilde{f}_{1}(t), \ldots, \tilde{f}_{d}(t)$ достаточно близки к $f_{1}(t), \ldots, f_{d}(t)$, то $F\left(\tilde{f}_{1}(t), \ldots, \tilde{f}_{d}(t)\right)$ тоже будет принадлежать $A[[t]]$ и будет иметь тот же свободный член. Таким образом, можно обеспечить, чтобы любой конкретный однородный элемент подалгебры $B(0)$, 
а значит, и любое конечное множество таких элементов принадлежали $\widetilde{B}(0)$. В частности, можно обеспечить, чтобы образующие подалгебры $B(0)$ принадлежали $\widetilde{B}(0)$; но тогда и вся подалгебра $B(0)$ будет содержаться в $\widetilde{B}(0)$ и из равенства рядов Пуанкаре будет следовать, что $B(0)=\widetilde{B}(0)$.

Замечание 1. Формальный предел свободной подалгебры может не быть свободной подалгеброй. Например, формальным пределом свободной подалгебры

$$
B(t)=K((t))\left[x^{2}+t y^{2}, x^{3}\right] \subset K((t))[x, y]
$$

является, как легко видеть, подалгебра

$$
B(0)=K\left[x^{2}, x^{3}, x^{4} y^{2}\right] \subset K[x, y],
$$

не являющаяся свободной.

Замечание 2. Формальный предел конечно порожденной подалгебры может не быть конечно порожденной подалгеброй. Например, формальным пределом подалгебры

$$
B(t)=K((t))\left[x+t y, x y, x y^{2}\right] \subset K((t))[x, y]
$$

является подалгебра

$$
B(0)=K\left[x, x y, x y^{2}, x y^{3}, \ldots\right] \subset K[x, y] .
$$

Однако мне неизвестно, может ли формальный предел свободной подалгебры не быть конечно порожденной подалгеброй.

Замечание 3. Мне также неизвестно, верно ли и в случае поля положительной характеристики, что в пространстве всех наборов однородных многочленов $f_{1}(t), \ldots, f_{d}(t) \in A((t))$ заданных степеней алгебраически независимые наборы образуют подмножество, открытое в формальной топологии.

Из предыдущего вытекает

Теорема 1. Формальный предел $B(0)$ любой однородной подалгебръь $B(t) \subset$ $A((t))$ имеет ту жее степень трансцендентности (над $K)$, что и $B(t)$ (над $K((t)))$.

Предполагая, что $K$ - поле нулевой характеристики, дадим более конструктивное доказательство этой теоремы.

Доказательство. Рассмотрение младших членов по $t$ показывает, что прообразы в $B(t) \cap A[[t]]$ алгебраически независимых элементов алгебры $B(0)$ алгебраически независимы в $B(t)$. Следовательно, tr. $\operatorname{deg} B(0) \leqslant \operatorname{tr} . \operatorname{deg} B(t)$.

Пусть tr. $\operatorname{deg} B(t)=d$, и пусть $f_{1}(t), \ldots, f_{d}(t) \in B(t)$ - алгебраически независимые однородные многочлены. Умножая их на подходящие степени $t$, можно добиться того, чтобы они лежали в $A[[t]]$. Пусть $J(t)$ - их якобиева матрица (относительно $\left.x_{1}, \ldots, x_{n}\right)$. Существует минор порядка $d$ этой матрицы, не равный нулю. Если он не делится на $t$, то матрица $J(0)$, являющаяся якобиевой матрицей многочленов $f_{1}(0), \ldots, f_{d}(0)$, имеет ранг $d$ и, значит, многочлены $f_{1}(0), \ldots, f_{d}(0)$ алгебраически независимы и $\operatorname{tr} . \operatorname{deg} B(0)=d$.

Если все миноры порядка $d$ матрицы $J(t)$ делятся на $t$, то многочлены $f_{1}(0), \ldots, f_{d}(0)$ алгебраически зависимы. Пусть

$$
F\left(f_{1}(0), \ldots, f_{d}(0)\right)=0
$$


- алгебраическая зависимость наименьшей степени между ними. Пусть для определенности многочлен $f_{d}(0)$ фактически участвует в этой зависимости, т. е. $\partial_{d} F \neq 0$. В силу нашего выбора многочлена $F$ имеем

$$
\left(\partial_{d} F\right)\left(f_{1}(0), \ldots, f_{d}(0) \neq 0 .\right.
$$

Это означает, что многочлен $F\left(f_{1}(t), \ldots, f_{d}(t)\right)$ делится на $t$ в $A[[t]]$, а многочлен $\left(\partial_{d} F\right)\left(f_{1}(t), \ldots, f_{d}(t)\right)$ не делится.

Заменив многочлен $f_{d}(t)$ на

$$
\tilde{f}_{d}(t)=t^{-1} F\left(f_{1}(t), \ldots, f_{d}(t)\right),
$$

мы снова получим алгебраически независимую систему многочленов из $B(t) \cap$ $A[[t]]$. Ее якобиева матрица $\tilde{J}(t)$ получается из $J(t)$ умножением последней строки на $t^{-1}\left(\partial_{d} F\right)\left(f_{1}(t), \ldots, f_{d}(t)\right)$ и прибавлением к ней некоторой линейной комбинации предыдущих строк, а миноры порядка $d$ матрицы $\tilde{J}(t)$ получаются из соответствующих миноров матрицы $J(t)$ умножением на $t^{-1}\left(\partial_{d} F\right)\left(f_{1}(t), \ldots\right.$, $\left.f_{d}(t)\right)$. Следовательно, наибольшая степень $t$, на которую делятся все миноры порядка $d$ якобиевой матрицы, при такой операции уменьшается на 1. Повторив эту операцию несколько раз, мы в конце концов придем к рассмотренной выше ситуации, когда в матрице $J(t)$ имеется минор порядка $d$, не делящийся на $t$.

\section{§2. Пределы подалгебр Мищенко-Фоменко}

Пусть $\mathfrak{g}$ - редуктивная алгебра Ли над полем $K$ нулевой характеристики и $P(\mathfrak{g})=\bigoplus_{m=0}^{\infty} P_{m}(\mathfrak{g})$ - ее алгебра Пуассона. Для каждого регулярного элемента $a \in \mathfrak{g}$ будем обозначать через $C(a)$ соответствующую подалгебру МищенкоФоменко алгебры $P(\mathfrak{g})$ (см. введение). Это коммутативная однородная подалгебра степени трансцендентности, равной размерности $b(\mathfrak{g})$ борелевской подалгебры алгебры $\mathfrak{g}$.

Пространство $C_{k}(a)$ однородных элементов степени $k$ алгебры $C(a)$ состоит из $k$-х дифференциалов инвариантов присоединенной группы в точке $a$. В частности, $C_{1}(a)$ совпадает с централизатором $\mathfrak{z}(a)$ элемента $a$ в алгебре $\mathfrak{g}$, размерность которого равна рангу алгебры $\mathfrak{g}$. (Напомним, что мы отождествляем $P_{1}(\mathfrak{g})=\mathfrak{g}^{*}$ с $\left.\mathfrak{g}.\right)$ Для описания пространства $C_{2}(a) \subset P_{2}(\mathfrak{g})$ удобно интерпретировать элементы пространства $P_{2}(\mathfrak{g})$ как симметрические операторы в $\mathfrak{g} ;$ тогда [3, теорема 2.1]

$$
C_{2}(a)=\left\{S \in P_{2}(\mathfrak{g}): \operatorname{ad}(a) S=\operatorname{ad}(b) \text { для некоторого } b \in \mathfrak{z}(a)\right\} .
$$

Заметим, что из равенства $\operatorname{ad}(a) S=\operatorname{ad}(b)$ (при условии, что $S-$ симметрический оператор) следует, что $S$ коммутирует с $\operatorname{ad}(a)$. При $b=0$ таким образом получается подпространство $C_{1}(a)^{2} \subset C_{2}(a)$. Его коразмерность в $C_{2}(a)$ равна полупростому рангу алгебры $\mathfrak{g}$.

Пример 1. Пусть $\mathfrak{g}=\mathfrak{g l}_{n}(K)$. Матрица $A$ является регулярным элементом алгебры $\mathfrak{g}$ тогда и только тогда, когда ее минимальный многочлен имеет степень $n$. (В этом случае матрицы $E, A, A^{2}, \ldots, A^{n-1}$ составляют базис ее централизатора $\mathfrak{z}(A)$.) Предположим, что матрица $A$ регулярна в этом смысле и невырожденна. Тогда $\mathfrak{z}(A) \ni A^{-1}$ и уравнение

$$
\operatorname{ad}(A) S=\operatorname{ad}\left(A^{-1}\right)
$$


имеет решение

$$
S: X \mapsto-A^{-1} X A^{-1}
$$

причем $S$ - симметрический оператор в алгебре $\mathfrak{g}$. Так как матрица $A^{-1}$ может быть любой регулярной невырожденной матрицей, то оператор $X \mapsto P X P$ является интегрируемым квадратичным гамильтонианом для любой регулярной невырожденной матрицы $P$. Отметим, что соответствующая квадратичная форма на $\mathfrak{g}$ имеет вид $\operatorname{tr}(P X)^{2}$.

Наряду с алгеброй $\mathfrak{g}$ будем рассматривать алгебру

$$
\mathfrak{g}((t))=\mathfrak{g} \otimes K((t)) .
$$

Это редуктивная алгебра Ли над полем $K((t))$, и мы можем применить метод сдвига инвариантов к ней. А именно, для любого регулярного элемента $a(t) \in$ $\mathfrak{g}((t))$ определена подалгебра Мищенко-Фоменко

$$
C(a(t)) \subset P(\mathfrak{g}((t)))=P(\mathfrak{g})((t)) .
$$

Это коммутативная однородная подалгебра, степень трансцендентности которой равна $b(\mathfrak{g}((t)))=b(\mathfrak{g})$, и можно рассмотреть ее формальный предел. Допуская некоторую вольность, мы будем обозначать его через $C(a(0))$. Очевидно, что это коммутативная однородная подалгебра алгебры $P(\mathfrak{g})$. Согласно теореме 1 , ее степень трансцендентности также равна $b(\mathfrak{g})$.

Заметим, что подалгебра $C(a(t))$ не изменяется при умножении $a(t)$ на любую степень $t$. Поэтому можно считать, что $a(t)$ - формальный степенной ряд (с коэффициентами из $\mathfrak{g}$ ) с ненулевым свободным членом.

Если ряд $F(t) \in \mathfrak{g}[[t]]$ принадлежит некоторой подалгебре Мищенко-Фоменко $C(a(t)) \subset P(\mathfrak{g}((t)))$, то его свободный член $F(0)$ по определению принадлежит ее формальному пределу $C(a(0)) \subset P(\mathfrak{g})$ и тем самым является интегрируемым гамильтонианом на алгебре $\mathfrak{g}$.

Замечание 4. Из предложения 1 и соображений, использованных при его доказательстве, следует, что если предельная подалгебра $C(a(0))$ является конечно порожденной, она не изменится при замене ряда $a(t)$ его достаточно большим начальным отрезком. При этом, если $K=\mathbb{R}$ или $\mathbb{C}$, формальный предел будет совпадать с обычным пределом.

Замечание 5. Сингулярные элементы алгебры $\mathfrak{g}((t))$ образуют замкнутое алгебраическое подмногообразие, определенное над $K$. Поэтому, если элемент $a(t)$ этой алгебры - многочлен от $t$, его регулярность равносильна тому, что $a\left(t_{0}\right)$ - регулярный элемент алгебры $\mathfrak{g}$ при некотором $t_{0} \in K$. Аналогично, если $K=\mathbb{R}$ или $\mathbb{C}$ и $a(t)$ - степенной ряд, сходящийся в некоторой окрестности нуля, то регулярность элемента $a(t)$ алгебры $\mathfrak{g}((t))$ равносильна тому, что $a\left(t_{0}\right)$ - регулярный элемент алгебры $\mathfrak{g}$ при всех $t_{0}$ из достаточно малой проколотой окрестности нуля.

Пример 2. Пусть $\mathfrak{g}=\mathfrak{g l}_{n}(K)$ и $P \in \mathfrak{g}$ - любая матрица. Рассмотрим элемент $P(t)=P+t Q$ алгебры $\mathfrak{g}[t]$, где $Q$ - какая-нибудь невырожденная регулярная матрица. В двумерном пространстве $\left\{s_{0} P+t_{0} Q: s_{0}, t_{0} \in K\right\}$ невырожденные регулярные матрицы образуют непустое открытое в топологии Зарисского подмножество, инвариантное относительно умножений на ненулевые элементы 
поля $K$, т. е. дополнение к объединению конечного числа одномерных подпространств. Следовательно, все матрицы вида $P+t_{0} Q, t_{0} \in K$, за исключением конечного числа, регулярны и невырожденны. Это означает, что $P(t)-$ невырожденный регулярный элемент алгебры $\mathfrak{g}((t))$.

Согласно примеру 1 в применении к алгебре $\mathfrak{g}((t))$, квадратичный гамильтониан

$$
S(t): X(t) \mapsto P(t) X(t) P(t)
$$

принадлежит некоторой подалгебре Мищенко-Фоменко в $P(\mathfrak{g}((t)))$ и, следовательно, его свободный член

$$
S(0): X \mapsto P X P
$$

принадлежит ее предельной подалгебре и тем самым является интегрируемым квадратичным гамильтонианом на алгебре $\mathfrak{g}$.

Таким образом, симметрический оператор $S: X \mapsto P X P$ является интегрируемым квадратичным гамильтонианом на алгебре $\mathfrak{g}=\mathfrak{g l}_{n}(K)$ для любой матрицы $P$. Если $\operatorname{Ker} P \not \subset \operatorname{Im} P$ (т. е. в жордановой форме матрицы $P$ есть нулевая клетка) и $\mathrm{rk} P>1$, то этот гамильтониан не входит ни в какую подалгебру Мищенко-Фоменко.

В самом деле, предположим, что найдутся такая регулярная матрица $A$ и матрица $B$, что

$$
\operatorname{ad}(A) S=\operatorname{ad}(B) .
$$

Тогда $\mathfrak{z}(B) \supset \operatorname{Ker} S$; но легко видеть, что если $\operatorname{Ker} P \not \subset \operatorname{Im} P$, то $\operatorname{Ker} S$ порождает алгебру всех матриц (как ассоциативную алгебру). Следовательно, $\operatorname{ad}(B)=0$ и, значит, $\operatorname{Im} S \subset \mathfrak{z}(A)$. Однако нетрудно проверить, что если rk $P>1$, то подпространство $\operatorname{Im} S \subset \mathfrak{g l}_{n}(K)$ либо некоммутативно, либо (при $P^{2}=0$ ) имеет слишком большую размерность, чтобы содержаться в централизаторе регулярной матрицы.

Пример 3. Пусть $\mathfrak{g}$ - расщепимая простая алгебра Ли, $\mathfrak{h}$ - ее картановская подалгебра, $\alpha_{1}, \ldots, \alpha_{r}$ - простые корни, $e_{1}, \ldots, e_{r}-$ соответствующие корневые векторы, $f_{1}, \ldots, f_{r}$ - корневые векторы, отвечающие противоположным корням, и $h_{i}=\left[e_{i}, f_{i}\right]$. Мы будем предполагать, что векторы $e_{i}$ и $f_{i}$ нормированы таким образом, что $\alpha_{i}\left(h_{i}\right)=2$. Пусть, далее, $\delta-$ старший корень, $e_{\delta}$ и $e_{-\delta}$ - корневые векторы, отвечающие корням $\delta$ и $-\delta$, и $h_{\delta}=\left[e_{\delta}, e_{-\delta}\right]$, причем $\delta\left(h_{\delta}\right)=2$. Имеем $h_{\delta}=\sum_{i=0}^{r} m_{i} h_{i}$, где $m_{1}, \ldots, m_{r}$ - некоторые натуральные числа.

Положим

$$
a(t)=e_{-\delta}+t \sum_{i=0}^{r} e_{i} \in \mathfrak{g}[t], \quad b(t)=\sum_{i=0}^{r} m_{i} f_{i}+t e_{\delta} \in \mathfrak{g}[t] .
$$

Согласно известному результату Костанта [7], $a\left(t_{0}\right)$ и, аналогично, $b\left(t_{0}\right)-$ регулярные полупростые элементы алгебры $\mathfrak{g}$ при всех $t_{0} \in K, t_{0} \neq 0$. Это означает, что $a(t)$ и $b(t)$ - регулярные полупростые элементы алгебры $\mathfrak{g}((t))$. Кроме того, легко видеть, что $[a(t), b(t)]=0$. Пусть $S(t)$ - симметрический оператор в $\mathfrak{g}((t))$, удовлетворяющий уравнению

$$
\operatorname{ad}(a(t)) S(t)=\operatorname{ad}(b(t)) .
$$


Тогда соответствующий квадратичный гамильтониан принадлежит подалгебpe $C(a(t)) \subset P(\mathfrak{g}((t)))$, а квадратичный гамильтониан, соответствующий младшему коэффициенту $S$ ряда $S(t)$, принадлежит ее предельной подалгебре и, значит, является интегрируемым.

В случае $\mathfrak{g}=\mathfrak{s l}_{n}(K)$ имеем $b(t)=t a(t)^{-1}$, и одним из решений уравнения (2) является оператор

$$
S(t): x(t) \mapsto-t^{-1}(b(t) x(t) b(t))_{0},
$$

где индекс 0 обозначает проекцию на пространство матриц с нулевым следом (параллельно единичной матрице). Его младший член есть оператор

$$
S: x \mapsto-(f x f)_{0},
$$

где $f=\sum_{i=0}^{r} f_{i}$. (Все коэффициенты $m_{i}$ в данном случае равны 1.) Это по существу гамильтониан того типа, который был описан в предыдущем примере. Было бы интересно найти явный вид гамильтониана $S$ в общем случае, т. е. для произвольной расщепимой простой алгебры Ли $\mathfrak{g}$.

\section{§3. Многообразие интегрируемых квадратичных гамильтонианов}

В этом параграфе мы будем считать, что поле $K$ алгебраически замкнуто.

Пусть $\mathfrak{g}$ - редуктивная алгебра Ли над $K$ и $\mathfrak{g}^{\text {reg }}-$ открытое (в топологии Зарисского) подмножество ее регулярных элементов. Мы докажем, что все квадратичные гамильтонианы, входящие в подалгебры Мищенко-Фоменко $C(a), a \in \mathfrak{g}^{\mathrm{reg}}$, и их формальные пределы образуют неприводимое алгебраическое подмногообразие в $P_{2}(\mathfrak{g})$.

Пусть $\operatorname{Comm}(\mathfrak{g}) \subset \mathfrak{g} \times \mathfrak{g}-$ совокупность пар коммутирующих элементов алгебры $\mathfrak{g}$. Известно [8], что это неприводимое алгебраическое многообразие. Пересечение

$$
\operatorname{Comm}^{0}(\mathfrak{g})=\operatorname{Comm}(\mathfrak{g}) \cap\left(\mathfrak{g}^{\text {reg }} \times \mathfrak{g}\right)
$$

является его открытым подмножеством.

Обозначим через $M_{2}^{0}(\mathfrak{g})$ совокупность всех квадратичных гамильтонианов, входящих в подалгебры $C(a), a \in \mathfrak{g}^{\text {reg }}$, и положим

$$
M_{2}(\mathfrak{g})=\overline{M_{2}^{0}(\mathfrak{g})}
$$

(замыкание в топологии Зарисского в $\left.P_{2}(\mathfrak{g})\right)$. Согласно описанию квадратичных элементов подалгебр Мищенко-Фоменко, приведенному в предыдущем параграфе, $M_{2}^{0}(\mathfrak{g})$ есть проекция на $P_{2}(\mathfrak{g})$ алгебраического многообразия

$$
\mathfrak{M}_{2}^{0}(\mathfrak{g})=\left\{(a, b, S) \in \operatorname{Comm}^{0}(\mathfrak{g}) \times P_{2}(\mathfrak{g}): \operatorname{ad}(a) S=\operatorname{ad}(b)\right\} .
$$

(Здесь элементы пространства $P_{2}(\mathfrak{g})$ понимаются как симметрические операторы в g.) Заметим, что многообразие $\mathfrak{M}_{2}^{0}(\mathfrak{g})$ неприводимо, так как оно проектируется на неприводимое алгебраическое многообразие $\operatorname{Comm}^{0}(\mathfrak{g})$ со слоями, являющимися аффинными пространствами. Следовательно, замыкание его проекции на $P_{2}(\mathfrak{g})$, т. е. $M_{2}(\mathfrak{g})$, также является неприводимым алгебраическим многообразием, а $M_{2}^{0}(\mathfrak{g})$ густо в нем (т. е. содержит непустое открытое подмножество).

Следующая общая лемма показывает, что $M_{2}(\mathfrak{g})$ - это и есть совокупность квадратичных элементов всех формальных пределов подалгебр Мищенко-Фоменко. 
Лемма 1. Пусть $\varphi: X \rightarrow Y-$ морбизм неприводимых алгебраических многообразий над полем $K$. Тогда, если $x(t) \in X(K((t)) u \varphi(x(t))=y(t) \in Y(K[[t]])$, то $y(0) \in \overline{\varphi(X)}$. Обратно, для любой точки $y \in \overline{\varphi(X)}$ найдется такая точка $x(t) \in X(K((t)))$, что $\varphi(x(t))=y(t) \in Y(K[[t]])$ u $y(0)=y$.

Доказательство. Очевидно, что

$$
\varphi(X(L)) \subset \overline{\varphi(X)}(L)
$$

для любого поля $L \supset K$. Кроме того, если $y(t) \in \overline{\varphi(X)}(K[[t]])$, то $y(0) \in \overline{\varphi(X)}$. Отсюда следует первое утверждение леммы.

При доказательстве второго утверждения можно, во-первых, считать, что $\overline{\varphi(X)}=Y$, т. е. морфизм $\varphi$ доминантен, и, во-вторых, перейдя к подходящим аффинным картам, - что $X$ и $Y$ являются аффинными многообразиями. При этих предположениях из теоремы о продолжении гомоморфизмов следует, что существует такой дивизор $D \subset Y$, что

$$
\varphi(X(L)) \supset Y(L) \backslash D(L)
$$

для любого алгебраически замкнутого поля $L \supset K$ (в частности, для $L=K$ ).

Далее, если $\operatorname{dim} Y>1$, то существует многочлен $F \in K[Y]$, обращающийся в нуль в данной точке $y \in Y$, но не обращающийся тождественно в нуль ни на одной неприводимой компоненте дивизора $D$. Заменив тогда $Y$ на какуюнибудь неприводимую компоненту дивизора $F=0$, a $X$ - на подходящую неприводимую компоненту ее полного прообраза, можно понизить размерность. Таким образом, достаточно доказать лемму в случае, когда $Y$ - кривая. В этом случае дивизор $D$ состоит из конечного числа точек и $D(L)=D$ для любого поля $L \supset K$

Итак, пусть $Y-$ (аффинная) кривая. Рассмотрим ее нормализацию $\nu: \widetilde{Y} \rightarrow Y$. Пусть $\tilde{y} \in \widetilde{Y}$ - какой-нибудь прообраз точки $y$. Морфизм $\nu$ определяется вложением алгебры $K[Y]$ в алгебру $K[\widetilde{Y}]$. Последняя алгебра, в свою очередь, вкладывается в пополнение локальной алгебры точки $\tilde{y}$, которая, как известно, изоморфна $K[[t]]$. Тем самым мы получаем гомоморфизм $K[Y] \rightarrow K[[t]]$, т. е. некоторую точку $y(t) \in Y(K[[t]])$. Очевидно, что $y(0)=y$, но $y(t) \neq y$ и, значит, $y(t) \notin D$. Следовательно, $y(t) \in \varphi(X(L))$, где $L$ - алгебраическое замыкание поля $K((t))$, т. е. поле рядов Пюизо. Это означает, что $y(t)=\varphi(x(t))$, где $x(t) \in X\left(K\left(\left(t^{\frac{1}{q}}\right)\right)\right.$ для некоторого $q \in \mathbb{N}$. Заменив $t$ на $t^{q}$, мы получим требуемую точку из $X(K((t)))$.

В нашей ситуации лемма применяется к многообразиям $X=\mathfrak{M}_{2}^{0}(\mathfrak{g}), Y=$ $P_{2}(\mathfrak{g})$.

Найдем размерность многообразия $M_{2}(\mathfrak{g})$ для полупростой алгебры Ли $\mathfrak{g}$ ранга $r$. Для фиксированной картановской подалгебры $\mathfrak{h} \subset \mathfrak{g}$ обозначим через $M_{2}^{0}(\mathfrak{g}, \mathfrak{h})$ совокупность всех квадратичных гамильтонианов, входящих в подалгебры $C(a), a \in \mathfrak{h}^{\text {reg }}=\mathfrak{h} \cap \mathfrak{g}^{\text {reg }}$. Очевидно, что

$$
M_{2}^{0}(\mathfrak{g}, \mathfrak{h})=P_{2}(\mathfrak{h}) \times N_{2}^{0}(\mathfrak{g}, \mathfrak{h}),
$$

где $N_{2}^{0}(\mathfrak{g}, \mathfrak{h})$ состоит из симметрических операторов вида

$$
S(a, b): \mathfrak{h} \rightarrow 0, \quad e_{\alpha} \mapsto \frac{\alpha(b)}{\alpha(a)} e_{\alpha} \quad\left(a \in \mathfrak{h}^{\mathrm{reg}}, b \in \mathfrak{h}\right) .
$$


Ясно также, что

$$
\operatorname{dim} P_{2}(\mathfrak{h})=\frac{r(r+1)}{2}, \quad \operatorname{dim} N_{2}^{0}(\mathfrak{g}, \mathfrak{h})=2 r-1 .
$$

Так как полупростые элементы образуют плотное подмножество в $\mathfrak{g}$, то

$$
M_{2}(\mathfrak{g})=\overline{\operatorname{Ad}(G) M_{2}^{0}(\mathfrak{g}, \mathfrak{h})},
$$

где $G$ - связная алгебраическая группа, соответствующая алгебре Ли g. Отметим, что корневые векторы $e_{\alpha}$ и $e_{-\alpha}$ являются собственными векторами оператора $S(a, b)$ с одним и тем же собственным значением. Поэтому картановская подалгебра $\mathfrak{h}$ однозначно восстанавливается по оператору общего положения из $M_{2}^{0}(\mathfrak{g}, \mathfrak{h})$ как сумма одномерных собственных подпространств. Следовательно,

$$
\operatorname{dim} M_{2}(\mathfrak{g})=\operatorname{dim} M_{2}^{0}(\mathfrak{g}, \mathfrak{h})+\operatorname{dim} \mathfrak{g}-r=\operatorname{dim} \mathfrak{g}+\frac{r^{2}+3 r-2}{2} .
$$

Пример 4. Для $\mathfrak{g}=\mathfrak{s l}_{2}(K)$ получаем $\operatorname{dim} M_{2}(\mathfrak{g})=4$. Далее, всякий оператор $S \in M_{2}^{0}(\mathfrak{g}, \mathfrak{h})$ в этом случае действует на ортогональном дополнении $\mathfrak{h}^{\perp}$ к картановской подалгебре скалярно. Отсюда следует, что $M_{2}(\mathfrak{g})$ есть замыкание множества полупростых симметрических операторов, имеющих кратное собственное значение. Операторы $S \in M_{2}(\mathfrak{g})$ могут быть охарактеризованы тем, что $E, S, S^{2}$ линейно зависимы (т. е. степень минимального многочлена не больше 2). Помимо полупростых операторов, $M_{2}(\mathfrak{g})$ содержит операторы, имеющие единственное (трехкратное) собственное значение и жордановы клетки порядков 2 и 1.

Положим

Ясно, что

$$
M_{2}(\mathfrak{g}, \mathfrak{h})=\overline{M_{2}^{0}(\mathfrak{g}, \mathfrak{h})}, \quad N_{2}(\mathfrak{g}, \mathfrak{h})=\overline{N_{2}^{0}(\mathfrak{g}, \mathfrak{h})}
$$

$$
M_{2}(\mathfrak{g}, \mathfrak{h})=P_{2}(\mathfrak{h}) \times N_{2}(\mathfrak{g}, \mathfrak{h})
$$

и что $N_{2}(\mathfrak{g}, \mathfrak{h})$ есть $(2 r-1)$-мерное неприводимое подмногообразие в пространстве $D(\mathfrak{g}, \mathfrak{h})$ диагональных (в базисе из корневых векторов) симметрических операторов в $\mathfrak{h}^{\perp}$. (Симметричность здесь означает равенство диагональных элементов, отвечающих противоположным корням.)

Пример 5. Для $\mathfrak{g}=\mathfrak{s l}_{2}(K)$ очевидно, что $N_{2}(\mathfrak{g}, \mathfrak{h})=D(\mathfrak{g}, \mathfrak{h})$. Отметим, однако, что уже в этом случае многообразие $M_{2}(\mathfrak{g})$ содержит операторы, сохраняющие $\mathfrak{h}$, но не принадлежащие $M_{2}(\mathfrak{g}, \mathfrak{h})$.

Пример 6. Для $\mathfrak{g}=\mathfrak{s l}_{3}(K)$ имеем $\operatorname{dim} N_{2}(\mathfrak{g}, \mathfrak{h})=\operatorname{dim} D(\mathfrak{g}, \mathfrak{h})=3$, так что $N_{2}(\mathfrak{g}, \mathfrak{h})=D(\mathfrak{g}, \mathfrak{h})$.

Пример 7. Для $\mathfrak{g}=\mathfrak{s l}_{4}(K)$ имеем $\operatorname{dim} N_{2}(\mathfrak{g}, \mathfrak{h})=5$, в то время как $\operatorname{dim} D(\mathfrak{g}, \mathfrak{h})$ $=6$, так что $N_{2}(\mathfrak{g}, \mathfrak{h})$ - гиперповерхность в $D(\mathfrak{g}, \mathfrak{h})$. Найдем ее уравнение.

Корневыми векторами в данном случае являются матричные единицы $E_{i j}$ $(i \neq j)$. Пространство $D(\mathfrak{g}, \mathfrak{h})$ состоит из операторов вида

$$
E_{i j} \mapsto p_{i j} E_{i j} \quad\left(p_{i j}=p_{j i} \in K\right) .
$$

Если $a=\operatorname{diag}\left(a_{1}, a_{2}, a_{3}, a_{4}\right), b=\operatorname{diag}\left(b_{1}, b_{2}, b_{3}, b_{4}\right)$, то для оператора $S(a, b)$, задаваемого формулой (4), имеем

$$
p_{i j}=\frac{b_{i}-b_{j}}{a_{i}-a_{j}} .
$$


Taк как $\operatorname{tr} a=0$, то матрица $a$ определяется числами

$$
a_{12}=a_{1}-a_{2}, \quad a_{23}=a_{2}-a_{3}, \quad a_{34}=a_{3}-a_{4} .
$$

Аналогично, матрица $b$ определяется числами

$$
b_{12}=b_{1}-b_{2}, \quad b_{23}=b_{2}-b_{3}, \quad b_{34}=b_{3}-b_{4} .
$$

Уравнения (5) переписываются в виде

$$
\begin{gathered}
b_{12}=p_{12} a_{12}, \quad b_{23}=p_{23} a_{23}, \quad b_{34}=p_{34} a_{34}, \\
b_{12}+b_{23}=p_{13}\left(a_{12}+a_{23}\right), \quad b_{23}+b_{34}=p_{24}\left(a_{23}+a_{34}\right), \\
b_{12}+b_{23}+b_{34}=p_{14}\left(a_{12}+a_{23}+a_{34}\right) .
\end{gathered}
$$

После исключения $b_{12}, b_{23}, b_{34}$ получаем равенства

$$
\begin{gathered}
p_{12} a_{12}+p_{23} a_{23}=p_{13}\left(a_{12}+a_{23}\right), \\
p_{23} a_{23}+p_{34} a_{34}=p_{24}\left(a_{23}+a_{34}\right), \\
p_{12} a_{12}+p_{23} a_{23}+p_{34} a_{34}=p_{14}\left(a_{12}+a_{23}+a_{34}\right),
\end{gathered}
$$

на которые можно смотреть как на систему однородных линейных уравнений относительно $a_{12}, a_{23}, a_{34}$. Эта система должна иметь ненулевое решение (так как $\left.a \in \mathfrak{h}^{\mathrm{reg}}\right)$. Приравнивая нулю ее определитель, после несложного вычисления получаем уравнение

$$
\sum_{(i, j, k, l)} \operatorname{sgn}(i, j, k, l) p_{i j} p_{j k} p_{k l}=0,
$$

где суммирование идет по всем перестановкам $(i, j, k, l)$ чисел $1,2,3,4$.

Многочлен от 6 переменных, стоящий в левой части этого уравнения, однороден по совокупности переменных и имеет по каждой из них степень 1 . Пользуясь этим и соображениями симметрии, легко доказать его неприводимость. Отсюда следует, что уравнение (6) задает в точности многообразие $N_{2}(\mathfrak{g}, \mathfrak{h})$.

В общем случае даже описание многообразия $N_{2}(\mathfrak{g}, \mathfrak{h})$ является сложной задачей. Кроме того, к этому не сводится описание многообразия $M_{2}(\mathfrak{g})$.

\section{ЛитеРАТУРА}

[1] Э. Б. Винберг, О некоторых коммутативных подалгебрах универсальной обертывающей алгебры, Изв. АН СССР, сер. матем., 54:1 (1990), 3-25.

[2] С. В. Манаков, Замечание об интегрировании уравнений Эйлера динамики $n$-мерного твердого тела, Функц. анализ и его прил., 10:4 (1976), 93-94.

[3] А. С. Мищенко, А. Т. Фоменко, Уравнения Эйлера на конечномерньх группах Ли, Изв. АН СССР, сер. матем., 42:2 (1978), 396-415.

[4] В. В. Шувалов, О пределах подалгебр Мищенко-Фоменко в алгебрах Пуассона полупростых алгебр Ли, Функц. анализ и его прил., 36:4 (2002), 55-64.

[5] W. Borho, H. Kraft, Über die Gelfand-Kirillov-Dimension, Math. Ann., 220:1 (1976), $1-24$.

[6] I. M. Gelfand, A. A. Kirillov, Sur les corps liés aux algèbres enveloppantes des algèbres de Lie, Inst. Hautes Études Sci. Publ. Math., 31 (1966), 5-19. 
[7] B. Kostant, The principal three-dimensional subgroup and the Betti numbers of a complex simple Lie group, Amer. J. Math., 81 (1959), 973-1032.

[8] R. W. Richardson, Commuting varieties of semisimple Lie algebras and algebraic groups, Compositio Math., 38:3 (1979), 311-327.

Московский государственный университет, механико-математический факультет

Поступило в редакцию e-mail: vinberg@zebra.ru 